\title{
Parametric study of the frequency-domain thermoreflectance technique
}

C. Xing', C. Jensen, Z. Hua, H. Ban', D. H. Hurley, M. Khafizov, and J. R. Kennedy

Citation: Journal of Applied Physics 112, 103105 (2012); doi: 10.1063/1.4761977

View online: http://dx.doi.org/10.1063/1.4761977

View Table of Contents: http://aip.scitation.org/toc/jap/112/10

Published by the American Institute of Physics

\section{Articles you may be interested in}

Spatially localized measurement of thermal conductivity using a hybrid photothermal technique

Journal of Applied Physics 111, 103505 (2012); 10.1063/1.4716474

Modeling optical absorption for thermoreflectance measurements

Journal of Applied Physics 119, 095107 (2016); 10.1063/1.4943176

Thermal conductivity measurements of non-metals via combined time- and frequency-domain thermoreflectance without a metal film transducer

Review of Scientific Instruments 87, 094902 (2016); 10.1063/1.4962711

A frequency-domain thermoreflectance method for the characterization of thermal properties

Review of Scientific Instruments 80, 094901 (2009); 10.1063/1.3212673

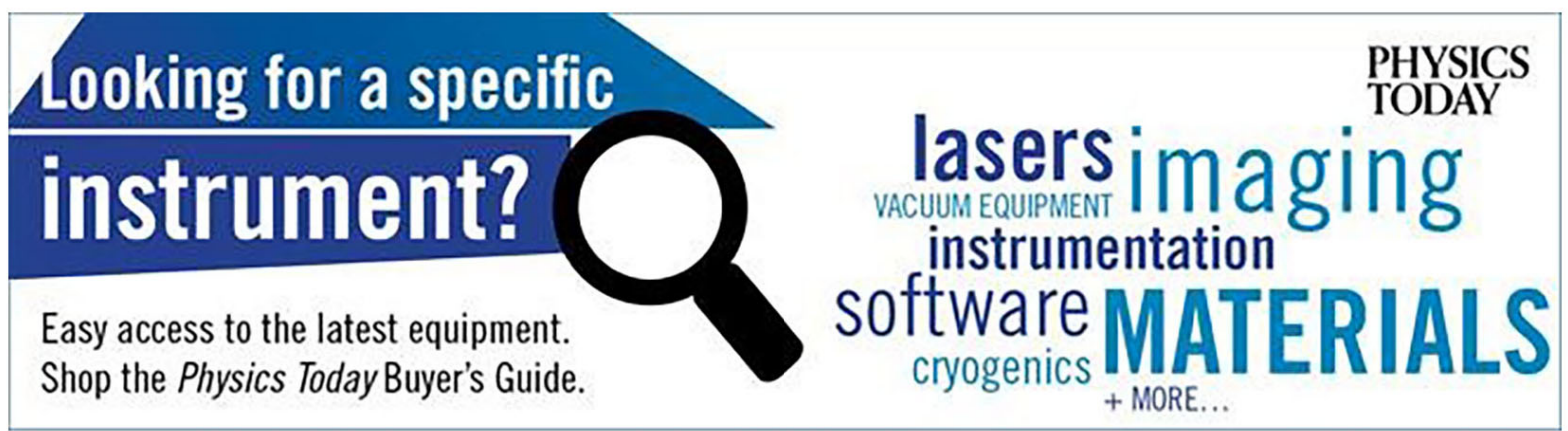




\title{
Parametric study of the frequency-domain thermoreflectance technique
}

\author{
C. Xing, ${ }^{1, a)}$ C. Jensen, ${ }^{1}$ Z. Hua,${ }^{1}$ H. Ban, ${ }^{1, a)}$ D. H. Hurley, ${ }^{2}$ M. Khafizov, ${ }^{2}$ and J. R. Kennedy ${ }^{2}$ \\ ${ }^{1}$ Department of Mechanical \& Aerospace Engineering, Utah State University, Logan, Utah 84322, USA \\ ${ }^{2}$ Idaho National Laboratory, Idaho Falls, Idaho 83415, USA
}

(Received 27 February 2012; accepted 3 October 2012; published online 21 November 2012)

\begin{abstract}
Without requiring regression for parameter determination, one-dimensional (1D) analytical models are used by many research groups to extract the thermal properties in frequency-domain thermoreflectance measurements. Experimentally, this approach involves heating the sample with a pump laser and probing the temperature response with spatially coincident probe laser. Micron order lateral resolution can be obtained by tightly focusing the pump and probe lasers. However, small laser beam spot sizes necessarily bring into question the assumptions associated with 1D analytical models. In this study, we analyzed the applicability of 1D analytical models by comparing to 2D analytical and fully numerical models. Specifically, we considered a generic nlayer two-dimensional (2D), axisymmetric analytical model including effects of volumetric heat absorption, contact resistance, and anisotropic properties. In addition, a finite element numerical model was employed to consider nonlinear effects caused by temperature dependent thermal conductivity. Nonlinearity is of germane importance to frequency domain approaches because the experimental geometry is such that the probe is always sensing the maximum temperature fluctuation. To quantify the applicability of the 1D model, parametric studies were performed considering the effects of: film thickness, heating laser size, probe laser size, substrate-to-film effusivity ratio, interfacial thermal resistance between layers, volumetric heating, substrate thermal conductivity, nonlinear boundary conditions, and anisotropic and temperature dependent thermal conductivity. (C) 2012 American Institute of Physics. [http://dx.doi.org/10.1063/1.4761977]
\end{abstract}

\section{INTRODUCTION}

Knowledge of basic thermophysical properties such as thermal conductivity and thermal diffusivity are essential for design and development of new materials and for inputs into numerical simulations. As a means of acquiring required thermal properties for different materials, geometries, and sizes, several measurement techniques have been developed over the past century. Examples of thermal conductivity measurement can be either direct, e.g., steady-state techniques such as the axial heat flow method ${ }^{1}$ and guarded hot plate method, ${ }^{2}$ or transient methods such as the hot wire, ${ }^{3}$ needle probe, ${ }^{4}$ and line source ${ }^{5}$ techniques; or indirect, e.g., the laser flash method ${ }^{6}$ that acquires thermal diffusivity, from which thermal conductivity may be calculated.

For multi-layered samples, photoacoustic and photothermal techniques have become extremely important measurement methods during the past 30 years because of their non-destructive, non-contact, fast, and accurate nature as well as high spatial resolution. Photothermal techniques include photothermal displacement, ${ }^{7}$ photothermal beam deflection (or "mirage"), ${ }^{8}$ photothermal radiometry (PTR) ${ }^{9}$ and photothermal reflectance (or thermoreflectance). ${ }^{10-13}$ Thermoreflectance techniques may be distinguished as one of the two measurement types: time-domain and frequencydomain measurements. In the frequency-domain thermoreflectance technique, a layered sample (an n-layer model is presented but the results are later presented for a single

\footnotetext{
a) Authors to whom correspondence should be addressed. Electronic addresses: changhu.xing@usu.edu and heng.ban@usu.edu.
}

layer on a substrate) is heated by the absorption of a modulated (sinusoidal) heat source supplied by a pump laser. Based on the temperature dependence of the sample's optical reflectance, the measurement system monitors the surface temperature response corresponding to the modulated heat source using a probe laser. By comparing the relationship between the modulated and reflected signals with theory, thermal property information of the sample structure is obtained. One advantage of the thermoreflectance technique over other photothermal methods is its high spatial resolution. By increasing the frequency of the heating laser and focusing the probe laser beam through an objective lens, this method is capable of quantitatively measuring the thermal effusivity of metals, glass, polymers, and ceramics coated with a metal film with spatial resolution near the optical diffraction limit $(\sim 1 \mu \mathrm{m})$. With a computer-controlled, motorized stage, local thermal effusivity maps of a sample surface can be obtained. ${ }^{11}$ To remove the complexity of lens alignments, fiber-aligned thermoreflectance is also under study. ${ }^{14}$

Precise modeling of the heat transport process in the layered-sample system is significant because the reflected signal only has an implicit relationship with the thermal properties of the system. Material properties or other parameters are extracted using an inverse method. When a 1D model is employed, the phase-effusivity relationship is determinate, which results in a simple data reduction process and a result with potentially lower uncertainty. Thus, this approach is preferred by many. ${ }^{10,11,13,15-17}$ However, the 1D model does not consider heat flow parallel to the layers; therefore, the range of applicability of the 1D model requires 
examination. To consider the effect of heat flow in the radial direction, Reichling and Gronbeck ${ }^{18}$ and Lepoutre et al. ${ }^{19}$ used three-dimensional (3D) (actually 2D axisymmetric) models and evaluated the influence of various parameters including film thickness, thermal conductivity, volume heating, and interfacial thermal resistance. However, several other important parameters warrant further analysis using the 2D axisymmetric model, especially when studying the use of the 1D model. The effusivity ratio of the layers is important for understanding the sensitivity of the system. The ratio of heating-spot size to thermal diffusion length is key to determining whether the use of a 1D model is appropriate or not. The ratio of pump to probe laser spot sizes plays an important role in the relationship between the phase taken from a single, center point vs. a finite probe size, which becomes increasingly important as the probe beam size approaches that of the pump beam. The effect of substrate thermal conductivity reveals frequency ranges in which even substrate thermal conductivity may be obtained from the results. Anisotropy of thermal conductivity in any layer of a multilayer system can cause strong deviation of results using an isotropic assumption. Furthermore, temperature-dependent thermal conductivity of the material makes the differential equation nonlinear and without analytical solution. The inclusion of temperature-dependent property in a numerical model is easily achievable, which can be used to study the measurement technique for nonlinear cases.

The n-layered material model has a specific advantage for irradiated samples. For nuclear fuels, the thermal property changes due to the change of material structure after irradiation. In neutron irradiated fuel, from the center to the exterior, to fuel rim, the microstructure may change drastically over a few millimeters. ${ }^{13}$ For ion-irradiated samples, a multi-layered structure may also be present due the possibility of a coating layer, damaged layer, ion implantation layer, and the original material. ${ }^{20}$ When measuring such samples, a multi-layered model may be used, which necessitates the derivation of an n-layered thermal model. For this preliminary work, however, a simpler, film-on-substrate model is studied.

The objective of this study is to analyze the influence of parameters on thermophysical property measurement using the thermoreflectance technique. In addition to the $1 \mathrm{D}$ and two-layered 2D axisymmetric models, this work derives an n-layer heat transfer model accounting for surface heating, volume heating, thermal contact resistance, etc., and compares results with a 2D numerical (finite element) analysis. Parametric studies reveal the conditions where the 1D model coincides with the 2D model. Each model can potentially be used in the data reduction process depending on the conditions of measurement.

\section{THEORETICAL MODELS}

Figure 1 presents an axisymmetric schematic of the thermoreflectance measurement of an n-layered sample. Due to axial symmetry, a cylindrical coordinate system is chosen with $\mathrm{Z}$-axis perpendicular to the sample surface and axially aligned with the laser spots. A thin film with a thickness, $\mathrm{L}_{1}\left(\mathrm{~L}_{\mathrm{i}}\right.$ is the

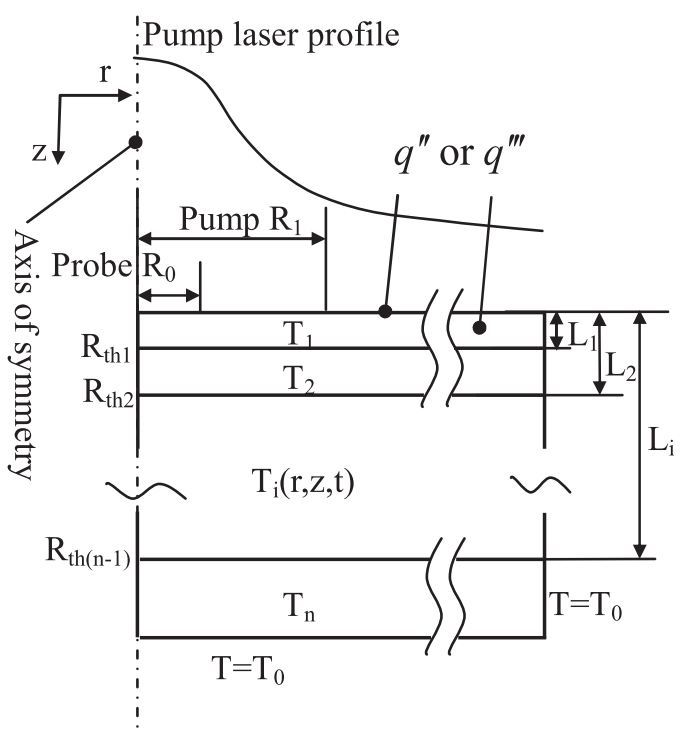

FIG. 1. Schematic illustration of n-layer domain and boundary conditions for thermoreflectance measurement.

distance of the ith layer from film surface $[\mathrm{m}])$, is coated on a substrate composed of one or more layers and considered thermally semi-infinite in both the radial, $r$, and the axial, $\mathrm{z}$ (last layer), directions. The intensity of the pump laser beam is assumed to have a Gaussian spatial distribution in the $\mathrm{r}$ direction. The heat supplied by the pump laser is absorbed either at the film surface (heat flux boundary condition) or in the volume of each layer (heat generation term in the energy equation).

Within each layer, the heat transport is assumed to be solely by conduction leading to the well-known energy equation in cylindrical coordinates including a source term

$$
\rho_{i} C_{p i} \frac{\partial T_{i}}{\partial t}=\frac{1}{r} \frac{\partial}{\partial r}\left(C_{k i} k_{i} r \frac{\partial T_{i}}{\partial r}\right)+\frac{\partial}{\partial z}\left(k_{i} \frac{\partial T_{i}}{\partial z}\right)+q_{i}^{\prime \prime \prime},
$$

where $T_{i}$ is the temperature distribution in space $(r, z$ directions) and evolution in time ( $[\mathrm{s}])[\mathrm{K}]$ and $\rho_{\mathrm{i}}, \mathrm{C}_{\mathrm{pi}}$, and $\mathrm{k}_{\mathrm{i}}$ are density $\left[\mathrm{kg} / \mathrm{m}^{3}\right]$, specific heat capacity $[\mathrm{J} /(\mathrm{kg} \mathrm{K})]$ and thermal conductivity $[\mathrm{W} /(\mathrm{m} \mathrm{K})]$ of the ith layer. If conductivity anisotropy (in-plane isotropic) is considered between the $\mathrm{r}$ and the $\mathrm{z}$ directions, $\mathrm{C}_{\mathrm{ki}}$ represents the $\mathrm{r}$ to $\mathrm{z}$ conductivity ratio for the ith layer. $q_{i}^{\prime \prime \prime}$ is the volumetric heat generation rate in each layer, $\left[\mathrm{W} / \mathrm{m}^{3}\right]$. Table I presents the reference values for the geometry and material properties of the two-layer system used in this work.

\section{A. 1D model}

For the common 1D phase model for a film/substrate sample, ${ }^{11}$ the derivatives with respect to the $r$ direction in

TABLE I. Geometry and thermal physical properties of the film on substrate system.

\begin{tabular}{lcc}
\hline \hline $\mathbf{L}_{\mathbf{1}}(\mu \mathrm{m})$ & $\mathbf{R}_{\mathbf{0 , 1}}(\mu \mathrm{m})$ & $\mathbf{P}(\mathrm{W})$ \\
1 & 5,15 & 0.01 \\
$\boldsymbol{\rho}_{\mathbf{1 , 2}}\left(\mathrm{kg} / \mathrm{m}^{3}\right)$ & $\mathbf{k}_{\mathbf{1 , 2}}(\mathrm{W} / \mathrm{m} \cdot \mathrm{K})$ & $\mathbf{C}_{\mathbf{p} \mathbf{1}, \mathbf{2}}(\mathrm{J} / \mathrm{kg} \cdot \mathrm{K})$ \\
7140,2220 & $34,1.38$ & 448,740 \\
\hline \hline
\end{tabular}


Eq. (1) and the $q_{i}^{\prime \prime \prime}$ are all zero. The heat flux boundary condition on the film surface is defined as

$$
-\left.k_{1} \frac{\partial T_{1}}{\partial z}\right|_{z=0}=q_{1}^{\prime \prime}[1+\sin (\varpi t)],
$$

where $q_{1}^{\prime \prime}$ represents heat flux applied in the 1D model $\left[\mathrm{W} / \mathrm{m}^{2}\right] . \omega$ is the angular speed of the pump beam function, $\omega=2 \pi \mathrm{f}[\mathrm{rad} / \mathrm{s}]$, and $\mathrm{f}$ is the modulated frequency $[\mathrm{Hz}]$.

The final expression of the 1D phase $(\delta[\mathrm{rad}])$ of the surface temperature from the heat flux in Eq. (2) is rewritten as

$$
-\delta=\frac{3}{4} \pi+\tan ^{-1} \frac{2 \cosh ^{2}(\gamma)[\tanh (\gamma)+\beta]\left[\tanh (\gamma)+\beta^{-1}\right]}{\sin (2 \gamma)\left(\beta-\beta^{-1}\right)} .
$$

An analysis of this equation indicates that of the five independent variables (laser frequency, f; film thickness, $\mathrm{L}_{1}$; film diffusivity, $\alpha_{1}\left(\alpha=\mathrm{k} /\left(\rho \mathrm{C}_{\mathrm{p}}\right)\left[\mathrm{m}^{2} / \mathrm{s}\right]\right)$; film effusivity, $\mathrm{e}_{1}$ $\left(\mathrm{e}=\left(\mathrm{k} \rho \mathrm{C}_{\mathrm{p}}\right)^{0.5},\left[\mathrm{~J} /\left(\mathrm{m}^{2} \mathrm{~K} \mathrm{~s}^{1 / 2}\right)\right]\right)$ and substrate effusivity, $\left.\mathrm{e}_{2}\right)$, only two are independent. $f, \alpha_{1}$, and $\mathrm{L}_{1}$, are related through variable $\gamma$ (the ratio of film thickness to thermal diffusion length, $\gamma=\mathrm{L}_{1} / \mathrm{L}_{\text {th }}$ and $\mathrm{L}_{\mathrm{th}}$ is the thermal diffusion/penetration length in terms of film material, $\left.\mathrm{L}_{\mathrm{th}}=\left[\alpha_{1} /(\pi \mathrm{f})\right]^{0.5},[\mathrm{~m}]\right)$. $\mathrm{e}_{1}$, and $\mathrm{e}_{2}$, are related through variable $\beta$ (the ratio of effusivities of substrate and film in the two layered system, $\beta=\mathrm{e}_{2} / \mathrm{e}_{1}$ ). If the film properties and thickness are known, the effusivity of substrate can be calculated.

\section{B. n-layer 2D axisymmetric model}

As an extension of the n-layer model for surface heating, ${ }^{21}$ the following derivation considers volume heating and interfacial thermal resistance between layers. In the literature, Li and Zhang ${ }^{12}$ have presented an n-layer volume heating model. The derivation method presented here is different, resulting in a distinct solution form. The derivation process is similar to that of Reichling and Gronbeck ${ }^{18}$ with generalization to n-layers. Two manners of heat deposition are considered, volumetric and surface heating, with each having either a heat source term in Eq. (1) or a heat flux boundary condition at the film surface.

When volumetric absorption is considered, the film surface is set to be adiabatic and the heat source term in Eq. (1) is expressed as

$$
\begin{gathered}
-\left.k_{1} \frac{\partial T_{1}}{\partial z}\right|_{z=0}=0, \\
q_{i}^{\prime \prime \prime}=\frac{2 P a_{i}}{\pi R_{1}^{2}} \prod_{k=1}^{i}\left(1-R_{f k}\right) e^{-\sum_{k=1}^{i-1} a_{k}\left(L_{k}-L_{k-1}\right)} e^{-2 r^{2} / R_{1}^{2}-a_{i}\left(z-L_{i-1}\right)+i \varpi t},
\end{gathered}
$$

where $a_{k}$ is the absorption coefficient of the each layer $\left[\mathrm{m}^{-1}\right], \mathrm{R}_{\mathrm{fk}}$ is the reflectivity, $\mathrm{P}$ is the pump laser power [W], and $R_{1}$ is the radius of pump laser spot based on a Gaussian distribution of intensity [m]. Typically, the coated film is selected to provide full absorption of the laser energy. Equation (5) is also a generalized n-layer form for the two layer absorption 18 .

Reference 22 uses a Gaussian distribution having a standard deviation defined differently than Eq. (5). (the $r$ distribution is characterized by $e^{-r^{2} / R_{1}^{2}}$ ). In Eq. (5), if the index $\mathrm{k}-1$ or $\mathrm{i}-1$ is less than 1 , then that term becomes zero.

An interfacial thermal resistance was considered in the 2D models, where across the interface, the heat flux is continuous but temperature has a discontinuity. The boundary condition across such an interface can be expressed as

$$
-\left.k_{i} \frac{\partial T_{i}}{\partial z}\right|_{z=L_{i}}=\frac{T_{i}-T_{i+1}}{R_{t h i}}=-\left.k_{i+1} \frac{\partial T_{i+1}}{\partial z}\right|_{z=L_{i}},
$$

where $R_{\text {thi }}$ is the thermal resistance between adjacent layers $\left[\mathrm{m}^{2} \mathrm{~K} / \mathrm{W}\right]$.

Assuming the temperature solution has a term of $e^{i \varpi t}$, application of the Hankel transformation, defined in Eq. (7), to all terms and boundary conditions reduces the 2D Eq. (1) to one dimensional ( $\mathrm{z}$ direction).

$$
\Delta \tilde{T}=\int_{0}^{\infty} r\left[T(r, z)-T_{0}\right] J_{0}(u r) d r
$$

where $\mathrm{u}$ is an auxiliary variable in Hankel transformation, $\mathrm{T}_{0}$ is the reference temperature from ambient $[\mathrm{K}], \mathrm{J}_{0}$ is the zeroth order Bessel function, and $\Delta \tilde{T}_{i}$ is the temperature change in transformed domain $[\mathrm{K}]$.

Equation (1) is transformed and reduced to

$$
\frac{\partial^{2} \Delta \tilde{T}_{i}}{\partial z^{2}}-\left(C_{k i} u^{2}+\frac{i \omega}{\alpha_{i}}\right) \Delta \tilde{T}_{i}=\tilde{q}_{i}^{\prime \prime \prime},
$$

where $\tilde{q}_{i}^{\prime \prime \prime}=\frac{P a_{i}}{2 \pi k_{i}} \prod_{k=1}^{i}\left(1-R_{f k}\right) e^{-\sum_{k=1}^{i-1} a_{k}\left(L_{k}-L_{k-1}\right)} e^{-a_{i}\left(z-L_{i-1}\right)-u^{2} R_{1}^{2} / 8}$. A solution to Eq. (8) can be written as

$$
\Delta \tilde{T}_{i}=C_{2 i-1} e^{-m_{i} z}+C_{2 i} e^{m_{i} z}+D_{i} e^{-a_{i}\left(z-L_{i-1}\right)}
$$

where $C_{2 i-1}$ and $C_{2 i}$ are auxiliary coefficients in the solution, $m_{i}=\sqrt{C_{k i} u^{2}+\frac{i \omega}{\alpha_{i}}}$ and

$$
D_{i}=\frac{-P a_{i} \prod_{k=1}^{i}\left(1-R_{f k}\right) e^{-\sum_{k=1}^{i-1} a_{k}\left(L_{k}-L_{k-1}\right)} e^{-u^{2} R_{1}^{2} / 8}}{2 \pi k_{i}\left[a_{i}^{2}-m_{i}^{2}\right]} .
$$

When a solution is sought, a relationship can be assumed for the two coefficients, $C_{2 i-1}$ and $C_{2 i}$, as $C_{2 i}=$ $F_{i} C_{2 i-1}+G_{i}\left(\mathrm{~F}_{\mathrm{i}}\right.$ and $\mathrm{G}_{\mathrm{i}}$ are intermediate coefficients). Using the interfacial boundary conditions, $F_{i}$ and $G_{i}$ can be written as 


$$
\begin{gathered}
F_{i}=\frac{\left(k_{i} m_{i}-k_{i+1} m_{i+1}+R_{t h i} k_{i} m_{i} k_{i+1} m_{i+1}\right) e^{-\left(m_{i}+m_{i+1}\right) L_{i}}+\left(k_{i} m_{i}+k_{i+1} m_{i+1}-R_{t h i} k_{i} m_{i} k_{i+1} m_{i+1}\right) F_{i+1} e^{\left(m_{i+1}-m_{i}\right) L_{i}}}{\left(k_{i} m_{i}+k_{i+1} m_{i+1}+R_{t h i} k_{i} m_{i} k_{i+1} m_{i+1}\right) e^{\left(m_{i}-m_{i+1}\right) L_{i}}+\left(k_{i} m_{i}-k_{i+1} m_{i+1}-R_{t h i} k_{i} m_{i} k_{i+1} m_{i+1}\right) F_{i+1} e^{\left(m_{i}+m_{i+1}\right) L_{i}}}, \\
G_{i}=\frac{\left[\left(k_{i} a_{i}-k_{i+1} m_{i+1}+R_{t h i} k_{i} a_{i} k_{i+1} m_{i+1}\right) D_{i} e^{-a_{i}\left(L_{i}-L_{i-1}\right)}+k_{i+1}\left(m_{i+1}-a_{i+1}\right) D_{i+1}\right] e^{-m_{i+1} L_{i}}+2 k_{i+1} m_{i+1} G_{i+1}}{\left(k_{i} m_{i}+k_{i+1} m_{i+1}+R_{t h i} k_{i} m_{i} k_{i+1} m_{i+1}\right) e^{\left(m_{i}-m_{i+1}\right) L_{i}}+\left(k_{i} m_{i}-k_{i+1} m_{i+1}-R_{t h i} k_{i} m_{i} k_{i+1} m_{i+1}\right) F_{i+1} e^{\left(m_{i}+m_{i+1}\right) L_{i}}} .
\end{gathered}
$$

$F_{i}$ and $G_{i}$ are functions of $F_{i+1}$ and $G_{i+1}$, which can be finally determined by the nth layer. To satisfy the finite reference temperature, $C_{2 n}$ needs to be zero so

$$
\begin{gathered}
F_{n-1}=\frac{\left(k_{n-1} m_{n-1}-k_{n} m_{n}+R_{t h(n-1)} k_{n-1} m_{n-1} k_{n} m_{n}\right) e^{-m_{n-1} L_{n-1}}}{\left(k_{n-1} m_{n-1}+k_{n} m_{n}+R_{t h(n-1)} k_{n-1} m_{n-1} k_{n} m_{n}\right) e^{m_{n-1} L_{n-1}},} \\
G_{n-1}=\frac{\left(k_{n-1} a_{n-1}-k_{n} m_{n}+R_{t h(n-1)} k_{n-1} a_{n-1} k_{n} m_{n}\right) D_{n-1} e^{-a_{n-1}\left(L_{n-1}-L_{n-2}\right)}+k_{n}\left(m_{n}-a_{n}\right) D_{n}}{\left(k_{n-1} m_{n-1}+k_{n} m_{n}+R_{t h(n-1)} k_{n-1} m_{n-1} k_{n} m_{n}\right) e^{m_{n-1} L_{n-1}}} .
\end{gathered}
$$

Applying the boundary condition on the first layer surface, the transformed temperature is

$$
\Delta \tilde{T}_{1}(0)=-\frac{D_{1} a_{1}}{m_{1}} \frac{1+F_{1}}{1-F_{1}}+\frac{2 G_{1}}{1-F_{1}}+D_{1} .
$$

For only one layer,

$$
\Delta \tilde{T}_{1}(0)=D_{1}\left(1-a_{1} / m_{1}\right) .
$$

For the surface heating condition, a heat flux is applied on the film surface. The heat source term in Eq. (1) is set to zero and the heat flux boundary condition can be expressed as

$$
-\left.k_{1} \frac{\partial T_{1}}{\partial z}\right|_{z=0}=\frac{2 P}{\pi R_{1}^{2}} e^{-2 r^{2} / R_{1}^{2}+i \varpi t} .
$$

Applying Hankel transformation, the flux boundary condition becomes

$$
-\left.k_{1} \frac{\partial \Delta \tilde{T}_{1}}{\partial z}\right|_{z=0}=\frac{P e^{-u^{2} R_{1}^{2} / 8}}{2 \pi}=q_{2}^{\prime \prime},
$$

where $q_{2}^{\prime \prime}$ is the heat flux $\left[\mathrm{W} / \mathrm{m}^{2}\right]$.

Similar to the volume heating case, a relationship can be assumed for the two coefficients, $C_{2 i}=F_{i} C_{2 i-1}$ and $\mathrm{G}_{\mathrm{i}}$ becomes zero. The $F_{i} s$ have the same form as those in Eqs. (10) and (12) and the transformed surface temperature can be written as

$$
\Delta \tilde{T}_{1}(0)=\frac{q_{2}^{\prime \prime}}{k_{1} m_{1}} \frac{1+F_{1}}{1-F_{1}} .
$$

For only one layer,

$$
\Delta \tilde{T}_{1}(0)=q_{2}^{\prime \prime} / k_{1} m_{1}
$$

Applying the inverse Hankel transformation, the periodic steady-state temperature at the surface $(z=0)$ is

$$
\Delta T_{1}(r, 0)=\int_{0}^{\infty} \Delta \tilde{T}_{1}(0) u J_{0}(r u) d u .
$$

The inverse Hankel transformation cannot be solved analytically and thus the inversion must be performed numerically. $\delta$ is the phase of the complex temperature, $\Delta \mathrm{T}_{1}$, $\tan ^{-1}\left[\operatorname{imag}\left(\Delta T_{1}(r, 0)\right) / \operatorname{real}\left(\Delta T_{1}(r, 0)\right)\right]$.

For an n-layer 1D model, the equation is identical to the transformed 2D equations without the " $u$ " term. Therefore, these coefficients are applicable for an n-layer 1D model as well.

The models from Reichling and Gronbeck and Lepoutre et al. are for two or three layers. Different from Li's derivation, ${ }^{12}$ this model is ideal for computation implemeting a loop structure, evidenced by considering the relationship between coefficients (Eq. (10) and (11)).

\section{2D numerical model}

Using the finite element software, COMSOL, a 2D axisymmetric model was created to represent the geometry in Fig. 1. 2D structured grids having an exponentially incremented spacing from the model axis and heating surface were used in the numerical simulations. Material properties and boundary conditions were applied according to Table I and those depicted in the n-layer analytical model, respectively. A thermal resistance may be added between the film and substrate using either a manually added, very thin layer or the software's built-in option, "thin thermally resistive layer." Grid and time-step independence tests were performed to show agreement with the analytical results.

The heat flux and/or heat generation was applied in the numerical model in a fashion similar to the forms presented in the previous sections. Transient computation was used to reach a periodic, steady-state condition (typically after 5-10 cycles) from which the temperatures of one to several cycles were used for data processing. Two methods were employed to find the amplitude and phase of the periodic temperature response of the sample. First, the difference of the maximum 
and minimum of the dataset provides peak-to-peak amplitude while a comparison of peak locations between the heat source (with a phase $\theta_{0}[\mathrm{rad}]$ ) and the sample provides the phase (lag). The second method is using Fourier analysis by means of integration with respect to sine and cosine $(\pi / 2$ phase difference) over the time period $(\Delta \mathrm{t},[\mathrm{s}])$. The amplitude is the modulus of $\frac{2}{\Delta t} \int_{\Delta t} \sin \left(\varpi t+\theta_{0}\right) T d t$ and $\frac{2}{\Delta t} \int_{\Delta t} \sin (\varpi t$ $\left.+\theta_{0}+\pi / 2\right) T d t$ and the phase is the angle between the two terms. The first approach requires manual attention while the second is applicable if the temperature peaks are nearly constant.

Although the 2D analytical model is sufficient for most purposes, the numerical model has advantages in handling more complicated descriptions of geometry, working conditions, and thermal properties. For instance, highly temperaturedependent thermal properties ${ }^{23}$ prevent analytical solutions, but are easily incorporated in the numerical model.

\section{RESULTS AND DISCUSSION}

As a simple introductory comparison of several models, Figure 2 presents temperature phase (a) and amplitude (b) as a function of modulation frequency of the heat source. Only surface heating was considered in this figure. The semi-

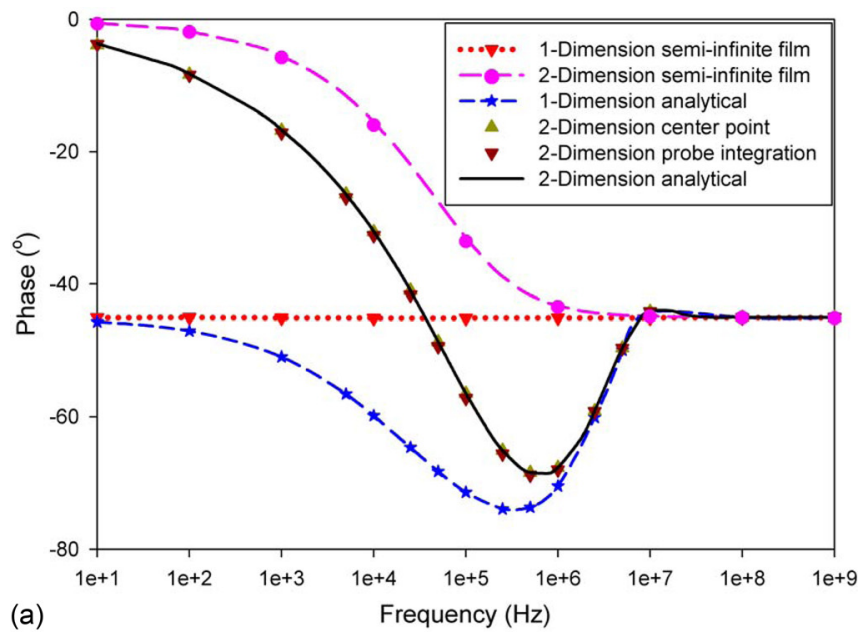

(a)

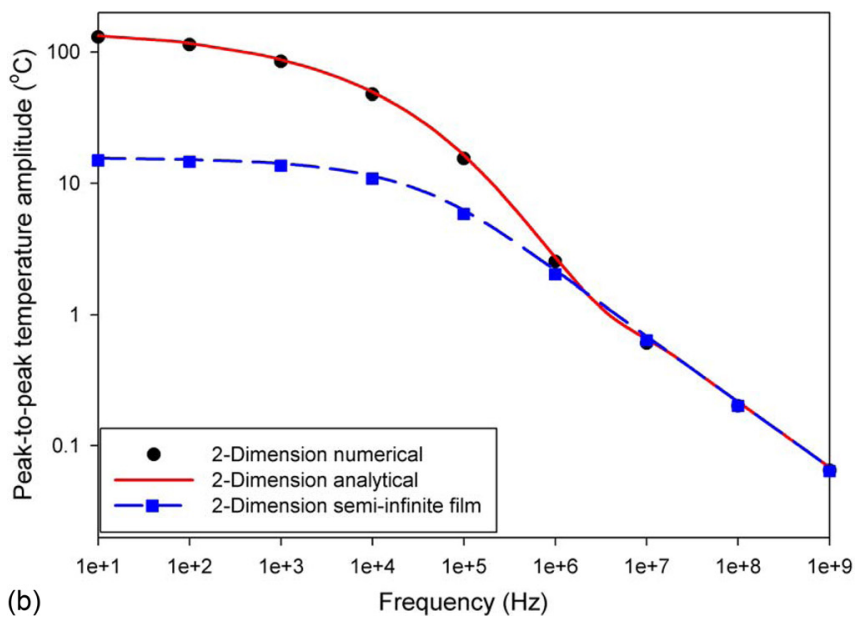

FIG. 2. Temperature phase and amplitude with respect to frequency for film/ substrate system. Lines - analytic results; Symbols - FEA results (a) phase and (b) amplitude. infinite film result is a case where the sample is homogeneous and composed of the film material only. The 1D model renders the well-known phase for a semi-infinite homogeneous material of $-45^{\circ}$ for all frequencies. However, for low frequencies and thus large $L_{t h}$ relative to $R_{1}$, the $2 D$ model shows deviation from the $1 \mathrm{D}$ model due to radial heat transfer. The phase from the $2 \mathrm{D}$ model is nearly synchronous with the heat source at low frequencies. Moving to higher frequencies, the phase asymptotically approaches $-45^{\circ}$, where $\mathrm{L}_{\text {th }}$ is much smaller than $\mathrm{R}_{1}$.

For the film/substrate system, the 1D phase is near $-45^{\circ}$ at both low and high frequencies because the heat transfer process is dominated by the substrate and film, respectively. At low frequencies (large $\mathrm{L}_{\mathrm{th}}$ ), the thermal wave penetrates deeply into the substrate; whereas at high frequencies (small $\mathrm{L}_{\mathrm{th}}$ ), the thermal waves stay within the film. Thus, in either case, the layered sample responds to the heat source essentially as a homogeneous sample. Through the intermediate frequency range, the phase deviates from $-45^{\circ}$ providing a frequency dependent response that can be used for parameter estimation.

The $2 \mathrm{D}$ results for the film/substrate system confirm the results from the 1D model at high frequencies. Whereas, at lower frequencies, the phase from 2D simulation is about $40^{\circ}$ less than those from the $1 \mathrm{D}$ model because the heat transfer is affected by $L_{1}$ and $R_{1}$ as will be demonstrated in Figure 4. For $\eta=3$ ( $\eta$ is the size ratio of pump laser spot to probe laser spot, $\eta=\mathrm{R}_{1} / \mathrm{R}_{0}$, and $\mathrm{R}_{0}$ is the probe laser radius based on Gaussian distribution of intensity [m]), the phases measured from a single point at the center of the probe spot and from the integration of temperature over the probe area do not differ significantly. In addition, the numerical and analytical results agree well with each other.

The peak-to-peak temperature amplitudes of the two cases in Figure 2(b) have similar trends except that the layered system has larger amplitude than the semi-infinite film material at low frequencies. This effect is due to the film diffusivity being much larger than that of the substrate, providing greater dissipation of temperature. When $\mathrm{L}_{\text {th }}$ becomes smaller than $\mathrm{L}_{1}$, the amplitudes show no difference. At high frequencies, the temperature amplitude is the classic result for a semi-infinite, homogeneous sample having $1 / \sqrt{f}$ dependency, a result of decreasing heat accumulation time.

For these particular sample and laser configurations, the use of the 1D model to investigate material properties is appropriate only for a frequency range $>5 \mathrm{MHz}$. However, because the $\mathrm{L}_{\text {th }}$ at $5 \mathrm{MHz}$ is already smaller than $\mathrm{L}_{1}$, a thinner film would be necessary to extract the desired information from the sample.

Figure 3 shows a comparison of phase responses for different film thicknesses $\left(\mathrm{L}_{1}\right)$. Modulation frequency was converted to the dimensionless number $\gamma$. With this relationship, all 1D results lie on the same curve. With decreasing $L_{1}$, the minimum frequency for which the 2D phase follows the 1D results also decreases. Physically, a reduction of film thickness reduces the proportion of radial diffusion in the film region thereby approaching a $1 \mathrm{D}$ description.

The phase response for the influence of heating laser size is presented in Fig. 4. In this figure, results are plotted for 


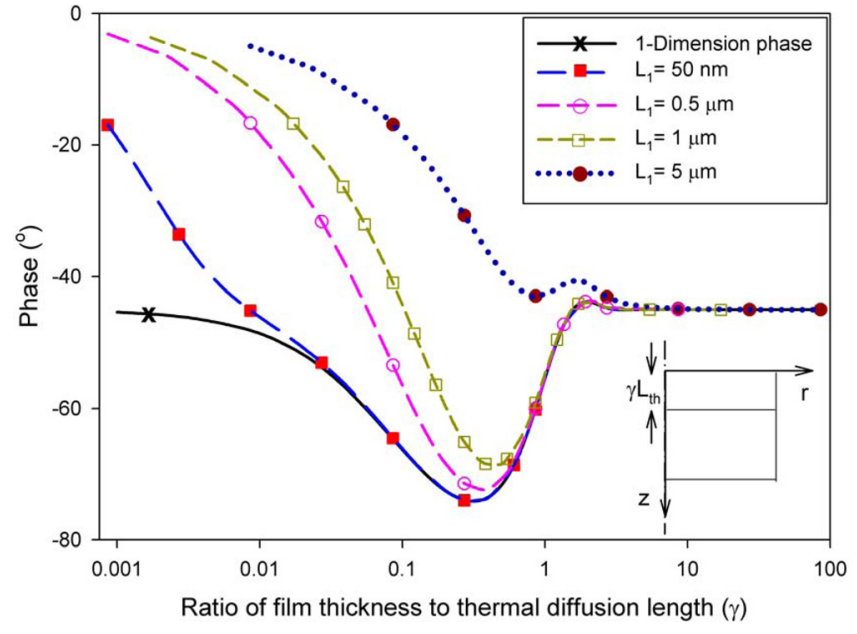

FIG. 3. Effect of film thickness on the phase response. Lines - analytic results; Symbols - FEA results.

several values of the dimensionless number $\lambda$, the ratio of $\mathrm{R}_{1}$ to $\mathrm{L}_{\mathrm{th}}\left(\lambda=\mathrm{R}_{1} / \mathrm{L}_{\mathrm{th}}\right)$. For $\mathrm{L}_{1}=0.1 \mu \mathrm{m}$, the phase differences between plane heating (1D) and point heating (2D) tend towards zero with increasing $\lambda$, throughout the entire frequency range. For a heating spot ten times $\mathrm{L}_{\mathrm{th}}$, the maximum error between $1 \mathrm{D}$ and $2 \mathrm{D}$ phases is only around $3 \%$ occurring near the phase minimum. However, when $\lambda$ is increased to 25 , the maximum error is only $0.3 \%$. Even when $\mathrm{L}_{1}$ is increased to $1 \mu \mathrm{m}$, the error is still less than $0.4 \%$. A large $\mathrm{R}_{1}$ relative to $\mathrm{L}_{\text {th }}$ will clearly provide a more uniform, 1D heat diffusion condition (plane heating in the probe area). This analysis quantitatively demonstrates the importance of $\lambda$ in deciding when the use of the 1D model is appropriate.

Figure 5 displays the percent deviation of phase obtained by temperatures integrated over the entire probe area from those taken from single-point temperatures $(\mathrm{r}=\mathrm{z}=0)$, as well as a comparison with the effective pump size method. ${ }^{19}$ $\mathrm{L}_{1}=100 \mathrm{~nm}$ is used in this figure. The maximum absolute deviation occurs in the intermediate frequency region; but the maximum relative deviation appears in the low frequency

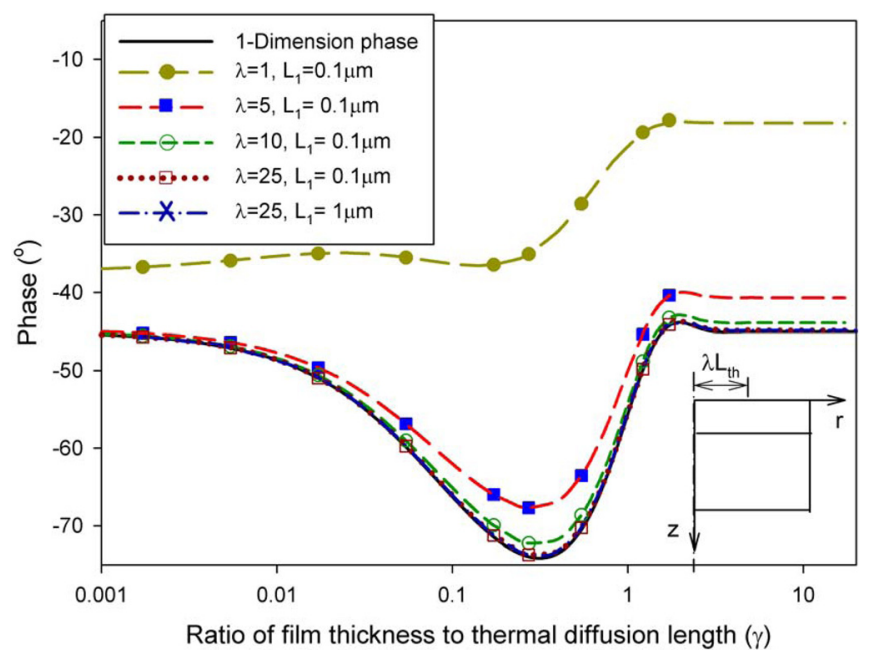

FIG. 4. Effect of heating laser radius on the phase response. Lines - analytic results; Symbols - FEA results

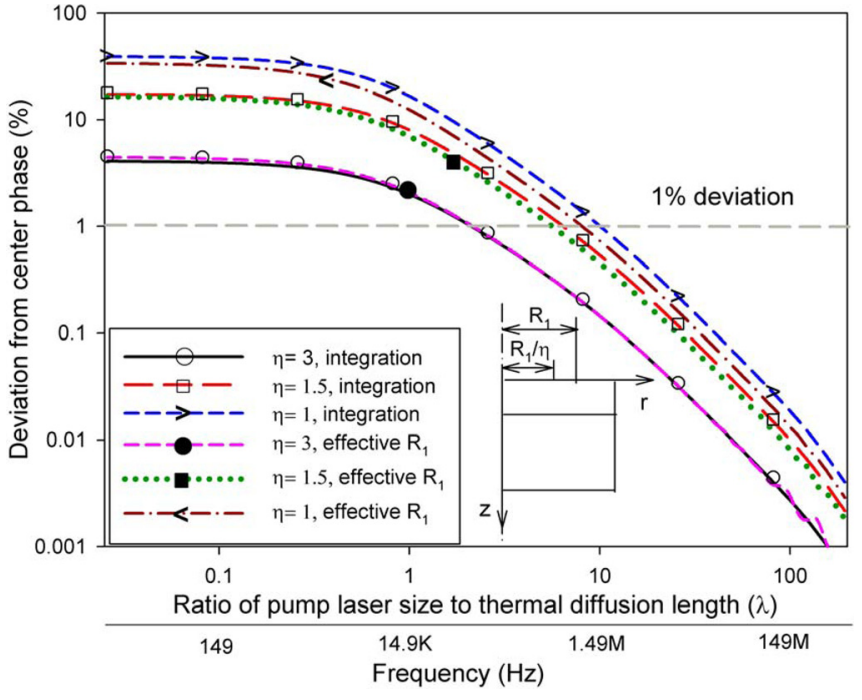

FIG. 5. Effect of using integration over probe area and effective pump laser size on the phase response. Lines - analytic results; Symbols - FEA results

range due to small absolute values (Figure 2(a)). The deviation increases with an increase of probe size ( $\operatorname{small} \eta$ ) especially in the low frequency range. However, the deviation becomes insignificant at high frequencies as $\mathrm{L}_{\text {th }}$ approaches or is less than $\mathrm{L}_{1}$. Figure 5 also shows the comparison to results using an effective pump laser size, $\sqrt{R_{0}^{2}+R_{1}^{2}}$. The use of an effective pump laser size is a simplified treatment used in place of the actual pump size to account for the pump/probe size relationship, while temperature is still taken only from the center point. If the probe beam is much smaller than the pump beam, the deviations from the two methods almost overlap. The difference between these two methods tends to increase with the increase of probe size. Figure 5 shows that for $\lambda>10-15$, the deviation associated with the integration process is less than $1 \%$. Thus, the probe size effect is consistent with the pump size effect.

For determining sample properties, effusivity ratio $(\beta)$ between film and substrate is the key parameter. Figure 6 presents a comparison of $1 \mathrm{D}$ and $2 \mathrm{D}$ phase responses for varying $\beta . \mathrm{L}_{1}=100 \mathrm{~nm}$ is used in this figure. From the $1 \mathrm{D}$ model, the effect of $\beta$ causes the phase to deviate from $-45^{\circ}$ creating a minima $\left(\delta<-45^{\circ}\right)$ for $0<\beta<1$ and a maxima $\left(\delta>-45^{\circ}\right)$ for $\beta>1$. The deviation is magnified for increasing/decreasing $\beta$ from 1 . However, the $2 \mathrm{D}$ model yields the phase close to zero for any $\beta$ in the low frequency range. At intermediate frequencies, the $2 \mathrm{D}$ results approach the $1 \mathrm{D}$ solution until overlapping it in the high frequency region. From a sensitivity point of view using the $1 \mathrm{D}$ solution $(\mathrm{d} \delta / \mathrm{d} \ln \beta),{ }^{24}$ low and high frequency ranges are less ideal for detection of $\beta$ since changes of $\ln \beta$ cause little variation in $\delta$. In the intermediate frequency range, the sensitivity reaches maximum when $\beta$ is close to unity and at a frequency where maximum phase occurs.

The influence of thermal resistance on phase is shown in Fig. 7 by the deviation of phase with resistance from that with perfect contact. With an increase of $\mathrm{R}_{\mathrm{th} 1}$, the phase in low and moderate frequency ranges is influenced more than at high frequency. An additional shift occurs in the moderate range 


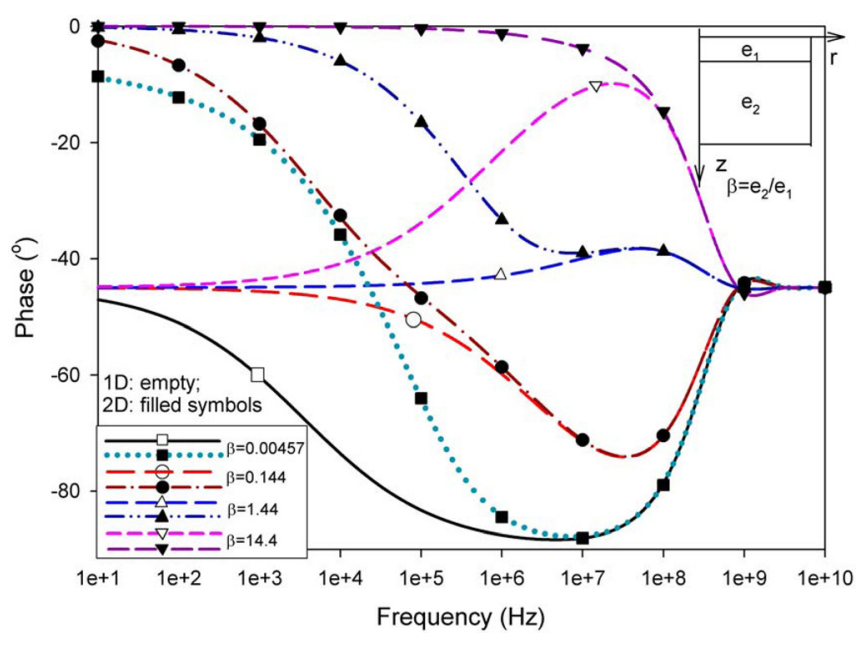

FIG. 6. Effect of effusivity ratio on the phase response. Lines - analytic results; Symbols - FEA results

because the effective thermal conductivity of the film/substrate system drops due to the increase of $\mathrm{R}_{\mathrm{th} 1}$ at the interface. The phase at low frequency is significantly affected by $3 \mathrm{D}$ heat transfer and $\mathrm{R}_{\mathrm{th} 1}$ but without any apparent trend. At high frequencies, the phase with thermal resistance approaches that of perfect contact due to the reduced $\mathrm{L}_{\text {th }}$, so that the thermal wave penetration is not deep enough to sense the interface. Clearly, detection and extraction of $\mathrm{R}_{\mathrm{th} 1}$ must be done using the intermediate frequency range because, in that range, the phase response is influenced by both layers with pronounced influence of $\mathrm{R}_{\mathrm{th} 1}$. The plots in Fig. 7 for different $\mathrm{L}_{1}$ (thinner film) clearly illustrate this idea. Further discussion and development regarding the influence of thermal resistance on such measurements may be found in Ref. 12 .

Figure 8 presents the effect of several types of volumetric heating vs. surface heating on phase response. $\mathrm{L}_{1}=100 \mathrm{~nm}$ is employed in this figure. For the case with only the first layer absorbing heat, the phase at moderate to high frequencies is strongly affected by the volumetric absorption of heat and increases in magnitude with an increase of absorption depth

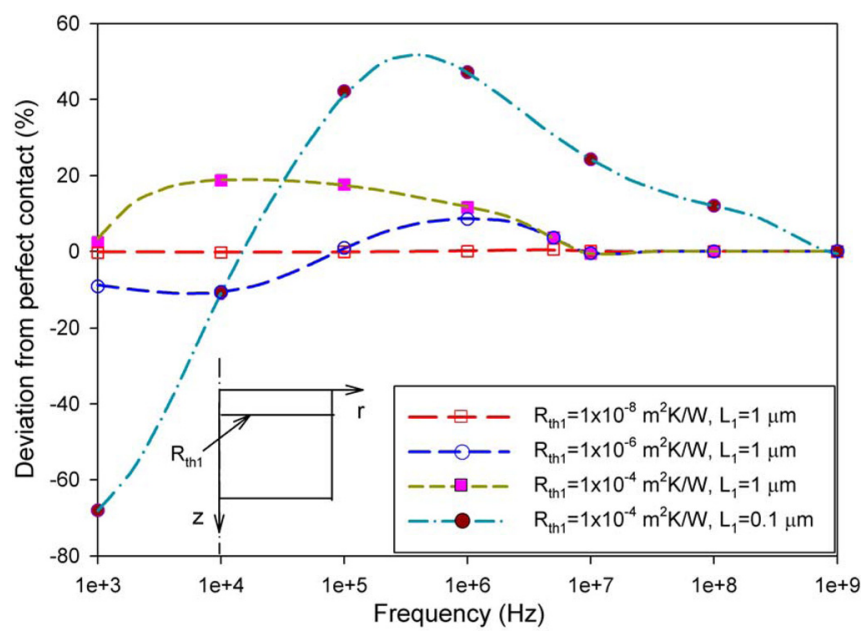

FIG. 7. Effect of interfacial thermal resistance on the phase response. Lines - analytic results; Symbols - FEA results

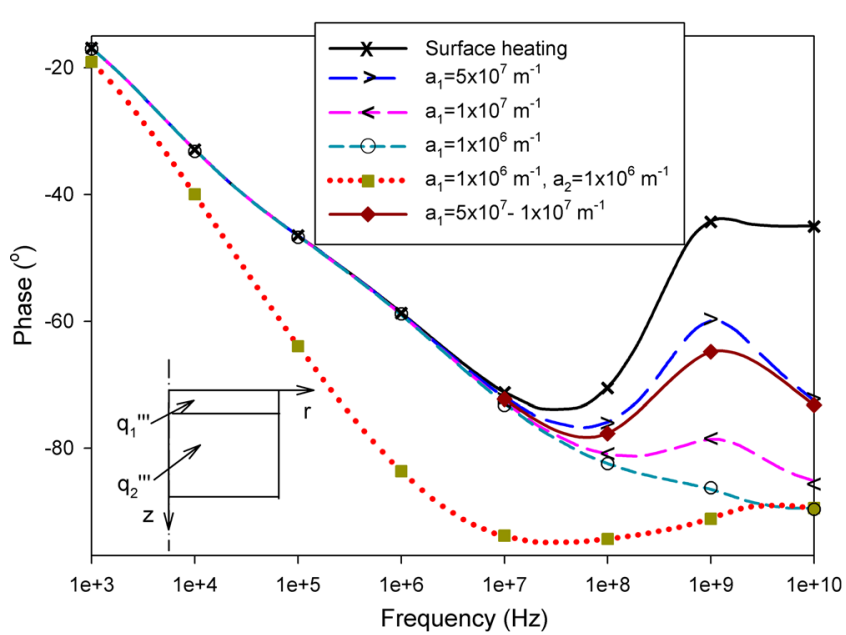

FIG. 8. Effect of absorption coefficient on the phase response. Lines - analytic results; Symbols - FEA results

$\left(\mathrm{a}_{1}^{-1}\right)$. At low frequencies, since $\mathrm{L}_{\mathrm{th}}$ is much larger than $\mathrm{a}_{1}{ }^{-1}$, the volumetric heating results approach those of surface heating only. On the contrary, in the high frequency range where $\mathrm{L}_{\text {th }}$ becomes much smaller than $\mathrm{a}_{1}{ }^{-1}$, the volumetric heating zone causes an additional phase shift approaching $-90^{\circ}$. If the $\mathrm{a}_{1}{ }^{-1}$ is much larger than $\mathrm{L}_{1}$ and the second layer also has a non-zero $a_{2}$, the result is a greatly modified phase even in the low frequency range, exceeding below the limiting value of $-90^{\circ}$ from the one layer volume heating. A case with linear depth-dependent $a_{1}$ from $5 \times 10^{7}$ at the surface to $1 \times 10^{7} \mathrm{~m}^{-1}$ at the interface is also superimposed on this figure. With a variable $a_{1}$, the phase response falls between those from the two constant $a_{1}$ cases. However, due to more pronounced heat influence from the surface, the response is closer to that with $\mathrm{a}_{1}=5 \times 10^{7} \mathrm{~m}^{-1}$. From inspection of a measured phase spectrum, indication may be found as to whether the material absorbs the heat at the surface or within the volume, by one or more layers. To appropriately employ the $1 \mathrm{D}$ model, the film material needs to be selected such that the heating is primarily at the film surface.

To consider the effect of substrate thermal conductivity, $\mathrm{k}_{2}$ was varied while keeping $\mathrm{e}_{2}$ the same. Figure 9 presents the effect of substrate thermal conductivity on the phase response (fixing e while changing $\mathrm{k}$ was done to magnify the effect of $\mathrm{k}$ at low frequency by maintaining similarity between different cases). For 1D modeling, substrate thermal conductivity (or diffusivity) does not participate in the calculation of phase. However, in the 2D model, this is only conditionally true. In Figure 9, while keeping other parameters the same, the following values are used: $\mathrm{R}_{\mathrm{th}}=1 \times 10^{-6} \mathrm{~m}^{2} \mathrm{~K} / \mathrm{W}$; $\mathrm{L}_{1}=0.1 \mu \mathrm{m}$ and surface and/or volume heating with $\mathrm{a}_{1}=1 \times 10^{7} \mathrm{~m}^{-1}$. At moderate to high frequencies, the value of $\mathrm{k}_{2}$ has no effect on the phase for either surface heating or volume heating. At low frequencies, the curves show distinction for different $\mathrm{k}_{2}$, independent of surface and/or volume heating. At low frequencies, the heat penetrates deeply into the substrate, dominating the phase response. Thus, with inclusion of $2 \mathrm{D}$ effects, the substrate thermal conductivity can potentially be found using low frequencies. Therefore, 


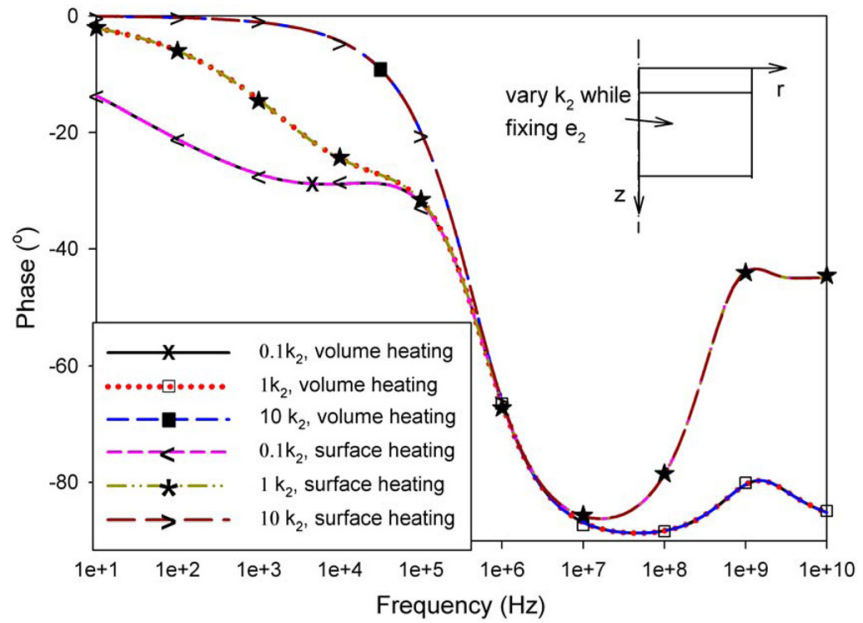

FIG. 9. Effect of substrate thermal conductivity while substrate effusivity remains constant on the phase response. Lines - analytic results; Symbols FEA results.

when $e_{2}$ is the target parameter, this frequency range should be used with caution.

Figure 10 presents results for anisotropic thermal conductivity in the radial (transverse isotropic) and axial directions. When $\mathrm{k}_{1}$ is increased by a factor of 10 in the radial direction, the resulting phase in the low and moderate frequency ranges increases from those of the isotropic model and deviates even more from the 1D solution. Similarly, when $\mathrm{k}_{2}$ is enlarged by a factor of 10 in the radial direction, phase shows similar behavior as anisotropic $\mathrm{k}_{1}$. The explanation for this effect is that increasing thermal conductivity in the radial direction accelerates radial thermal diffusion, thus becoming further from the 1D assumption of unidirectional heat flow. When the thermal conductivity in the radial direction is decreased, the radial heat conduction is lessened and more heat is transferred in the in-depth direction thus causing the phase response to be closer to the 1D results. In either case, the anisotropy effect in the film domain is more pronounced. Anisotropic effects are less pronounced at higher frequencies.

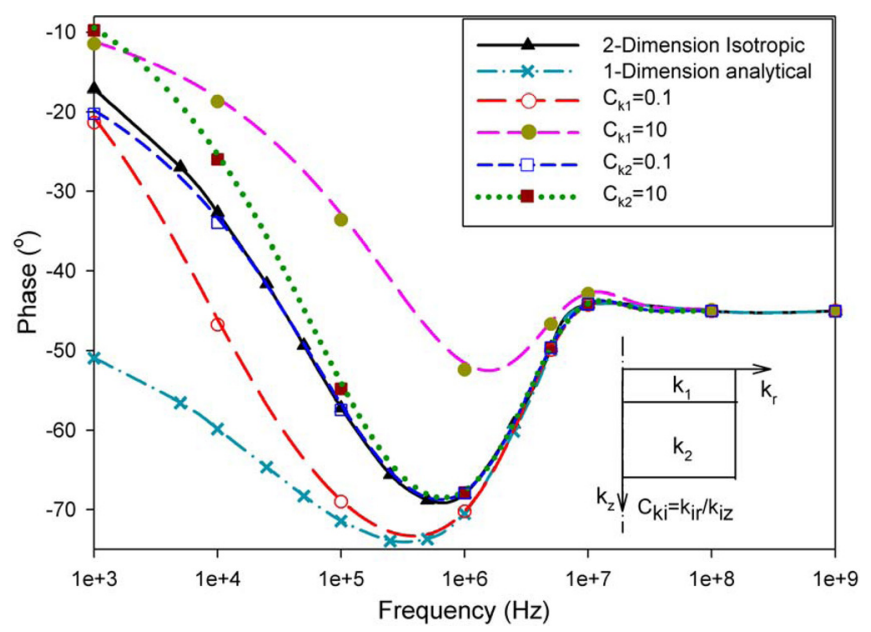

FIG. 10. Effect of anisotropic thermal conductivity on the phase response. Lines - analytic results; Symbols - FEA results.

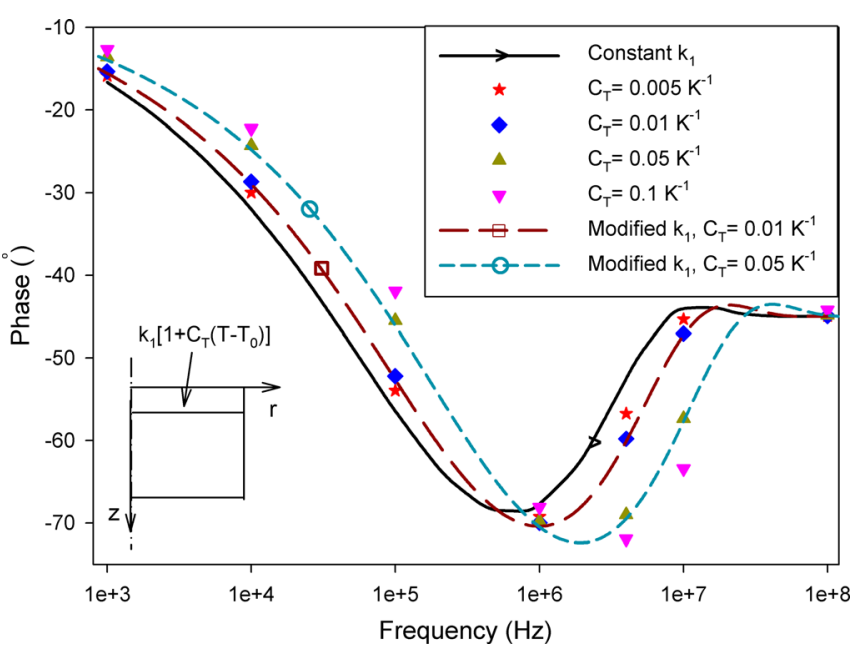

FIG. 11. Effect of temperature-dependent thermal conductivity on the phase response. Lines - analytic results; Symbols - FEA results.

To demonstrate an example of the power of the numerical simulation, a study on the effect of temperaturedependent thermal conductivity is presented in Fig. 11. In this figure, $\mathrm{k}_{1}$ is a function of temperature which includes temperature dependency, $\mathrm{k}_{1}\left[1+\mathrm{C}_{\mathrm{T}}\left(\mathrm{T}-\mathrm{T}_{0}\right)\right]$, where $\mathrm{C}_{\mathrm{T}}$ is a temperature dependent coefficient chosen as $0.005,0.01$, 0.05 , and $0.1 \mathrm{~K}^{-1}$ (for instance, stainless steel 304, Inconel or other amorphous materials have a positive $\mathrm{C}_{\mathrm{T}}$ ). The heat flux boundary condition was set in the form of $1+\sin (\omega t)$; but for each $\mathrm{C}_{\mathrm{T}}$ value used, the temperature field at $10 \mathrm{~s}$ was first achieved without the periodic term (after $0.1 \mathrm{~s}$, the center temperature approaches constancy). The periodic term was then engaged and the resulting phase at different frequencies was obtained by the Fourier transformation method with the 10th cycle. With increasing $\mathrm{C}_{\mathrm{T}}$, the resulting phase increasingly deviates from the case of constant thermal conductivity. However, for a constant thermal conductivity having a value modified according to the mean center temperature at $10 \mathrm{~s}$, the resulting phase is very close to that of the temperaturedependent situation. The largest deviation occurs at low frequencies by only $3 \%$ for $\mathrm{C}_{\mathrm{T}}=0.05$ and $1.5 \%$ for $\mathrm{C}_{\mathrm{T}}=0.01 \mathrm{~K}^{-1}$. The temperature dependency associated with the periodic terms seems to be averaged out over the cycle. In experiment, the measured phase for a temperature-dependent material is its value at the center mean temperature.

In the derivation of the analytical solution, convection and radiation heat transfer was neglected at the surface. To quantify their influence, convection and radiation were applied in the numerical model together with the heat flux boundary condition. Figure 12 presents percentage deviation of results with convection and radiation, from those with heat flux only. A moderate heat transfer coefficient $50 \mathrm{~W} /\left(\mathrm{m}^{2} \mathrm{~K}\right)$ was applied to account for convection; while $0.1,0.5$, and 0.9 were used for the emissivity of the sample surface. The maximum deviation is only $5 \%$ for $0.1 \mathrm{~Hz}$ and decreases exponentially with frequency. The deviation becomes less than $0.01 \%$ above $10 \mathrm{KHz}$ which guarantees the accuracy of the application of the model neglecting convection and radiation heat transfers. 


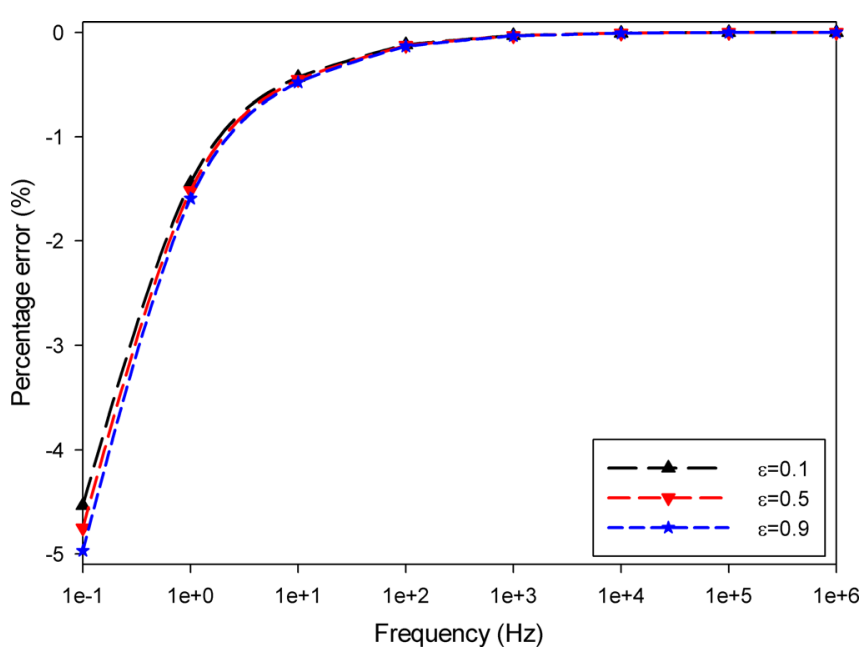

FIG. 12. Effect of convection and radiation boundary condition on the phase response.

\section{SUMMARY AND CONCLUSIONS}

1D analytical, 2D analytical, and 2D finite-element heat conduction models were described and used for a parametric study of the thermoreflectance technique. The n-layer, semiinfinite, 2D analytical model was derived in rigorous detail. A detailed comparison of a 1D and 2D models applied to a film/ substrate system was made to understand the influence of various model assumptions and parameters. The results provide useful guidance for system and sample design. They will also aid interpretation of experimental results as to which parameters should be included in the parameter estimation process and when the 1D model may be correctly applied.

The comparison of analytical results to the finite-element results shows good agreement in all cases. The finite-element model provides a powerful tool that may be used to investigate more complicated geometries or dependencies with minimal additional effort. For example, the effect of linearly temperature-dependent properties of a material has been shown to be equivalent to the case of a constant property value taken at the mean temperature of periodic heating.

\section{ACKNOWLEDGMENTS}

The work is supported by U.S. Department of Energy, Office of Nuclear Energy, under DOE Idaho Operations Office, Contract No. DE-AC07-05ID14517. Work by C.J. is supported using funding received from the DOE Office of Nuclear Energy's Nuclear Energy University Programs. D.H.H. and M.K. were supported as part of the Center for Materials Science of Nuclear Fuel, an Energy Frontier Research Center funded by the U.S. Department of Energy, Office of Science, Office of Basic Energy Sciences under Award No. 2009INL-FWP1356.

${ }^{1}$ ASTM E1225-04 Standard Test Method for Thermal Conductivity of Solids by Means of the Guarded-Comparative-Longitudinal Heat Flow Technique.

${ }^{2}$ ASTM C177-04 Standard Test Method for Steady-State Heat Flux Measurements and Thermal Transmission Properties by Means of the GuardedHot-Plate Apparatus.

${ }^{3}$ ASTM C1113M - 09 Standard Test Method for Thermal Conductivity of Refractories by Hot Wire (Platinum Resistance Thermometer Technique).

${ }^{4}$ ASTM D5334 - 08 Standard Test Method for Determination of Thermal Conductivity of Soil and Soft Rock by Thermal Needle Probe Procedure.

${ }^{5}$ ASTM D5930-01 Standard Test Method for Thermal Conductivity of Plastics by Means of a Transient Line-Source Technique.

${ }^{6}$ ASTM E1461-07 Standard Test Method for Thermal Diffusivity by the Flash Method.

${ }^{7}$ M. Soltanolkotabi et al., J. Appl. Phys. 85, 794 (1999).

${ }^{8}$ A. C. Boccara et al., Appl. Phys. Lett. 36, 130 (1980).

${ }^{9}$ M. Depriester et al., Rev. Sci. Instrum. 78, 036101 (2007).

${ }^{10} \mathrm{~N}$. Taketoshi et al., AIP Conf. Proc. 463, 315 (1999).

${ }^{11}$ K. Hatoria et al., Rev. Sci. Instrum. 76, 114901 (2005)

${ }^{12}$ B. C. Li and S. Y. Zhang, J. Phys. D: Appl. Phys. 30, 1447 (1997).

${ }^{13}$ Z. Hua et al., J. Appl. Phys. 111, 103505 (2012).

${ }^{14}$ J. A. Malen et al., J. Heat Transfer 133, 081601 (2011).

${ }^{15}$ R. Kato and I. Hatta, Int. J. Thermophys. 26, 179 (2005).

${ }^{16} \mathrm{H}$. Shibata et al., High Temp. Mater. Processes 29, 515 (2010). http:// eprints.iisc.ernet.in/35822/1/thermal.2010.pdf

${ }^{17}$ W. F. Bu et al., Thin Solid Films 516, 8359 (2008).

${ }^{18}$ M. Reichling and H. Gronbeck, J. Appl. Phys. 75, 1914 (1994).

${ }^{19}$ F. Lepoute et al., J. Appl. Phys. 78, 2208 (1995).

${ }^{20}$ H. Kyle et al., Mater. Sci. Eng., B 177, 164 (2012).

${ }^{21}$ D. G. Cahill, Rev. Sci. Instrum. 75, 5119 (2004).

${ }^{22}$ S. Volz, Microscale and Nanoscale Heat Transfer (Springer, 2007).

${ }^{23}$ M. Kuwahara et al., Microelectron. Eng. 84, 1792 (2007).

${ }^{24}$ A. J. Schmidt et al., Rev. Sci. Instrum. 80, 094901 (2009) 